\title{
Numerical Analysis Techniques for Wideband Amplifiers
}

\author{
Namkyoo Park, Won Jae Lee, Bumki Min, and Jaehyoung Park \\ Optical Communication Systems Laboratory \\ School of Electrical Engineering, Seoul National University, Seoul, Korea, 151-742 \\ Tel : +82-2-880-1820 Fax : +82-2-885-5284 E-mail : nkpark@plaza.snu.ac.kr
}

\begin{abstract}
Increasing demands on the high capacity wavelength division multiplexed (WDM) transmission system now require newly developed transmission windows beyond the gain bandwidth supported by erbium-doped fiber amplifiers (EDFA). With the intensive development efforts on new rare-earth dopants and fiber nonlinearity (Raman process) for fast few years, wideband optical amplifiers now can support easily over 4-5 fold wider gain bandwidth than it was formerly possible with the conventional EDFAs. Of various breeds for this application, there exist three distinct approaches near $1500 \mathrm{~nm}$ band, accessible in the commercial market. These includes : Thulium-doped fluoride fiber amplifiers (TDFFA) for S+ band (1450-1480 nm) and S band $(1480-1530 \mathrm{~nm})$, EDFAs for C band (1530-1560nm) and L band (1570-1610nm), Raman amplifiers with 100nm's of gain bandwidth (with flexible location from S+ to L band), and hybrid amplifiers with serial / parallel combination of above techniques. Even though there have been much increased experimental reports for all of these amplifiers, the complexity of the amplification dynamics from the number of involving energy levels and difficulty in measuring experimental parameters make it harder than ever to predict the performance of wideband amplifiers in general. This lack of serious study on the analytic or numerical analysis on wideband amplifiers could cause the future impairments for the prediction and estimation of the amplifier performances for different applications, restricting the successful deployment of wideband amplifier based transmission systems. In this paper, we present the numerical model and analysis techniques for wideband amplifiers (C/L band EDFA, Raman amplifier, and TDFA), along with their application examples.
\end{abstract}

Keywords : Erbium-doped fiber amplifiers (EDFAs), Thulium-doped fiber amplifiers (EDFAs), Raman amplifiers, Fiber amplifiers, wavelength division multiplexed (WDM) systems, optical fiber communication

\section{C+L BAND EDFA MODELING}

\subsection{Introduction}

Among many attempts suggested so far, the silica based EDFAs in parallel configuration (C-band: 1530 1560nm, plus L-band: $1570 \sim 1610 \mathrm{~nm}$ ) [1, 2] have been considered to be the most immediate viable solution for real system applications, from the maturity of the supporting technologies such as the host material and pump sources. Still, when compared to the C-band EDFA (C-EDFA), there have not existed corresponding accumulated research efforts on L-band EDFA (L-EDFA) except last few years. The most serious research focus on L-EDFA recently has been addressed to the efficiency improvement, to relax the requirement for much higher pump power when compared to C-EDFA. As one of the approaches to improve power conversion efficiency in L-band EDFAs [3, 4], we have suggested a structure which recycles useless backward amplified spontaneous emission (ASE) of L-EDFA as a secondary pumping source in the passive section of EDFA [4]. In this section, we provide spatially resolved W-EDFA numerical analysis result in detail, to clearly reveal the roles of the C-band injection source to the evolution dynamics of the primary pump, L-EDFA backward ASE, C-band injection sources and L-EDFA signals. As an application example, we investigate a novel W-EDFA structure, without the use of additional injection sources [5, 6]. Experimental results show considerable improvements on gain and noise figure $(2.6 \mathrm{~dB}$ and $0.6 \mathrm{~dB}$ at $3.5 \mathrm{dBminput}$ ) in addition to the limiting behavior, with negligible channel crosstalk from $\mathrm{C}$ to L-EDFAs. 


\subsection{Experiments / Numerical Analysis}

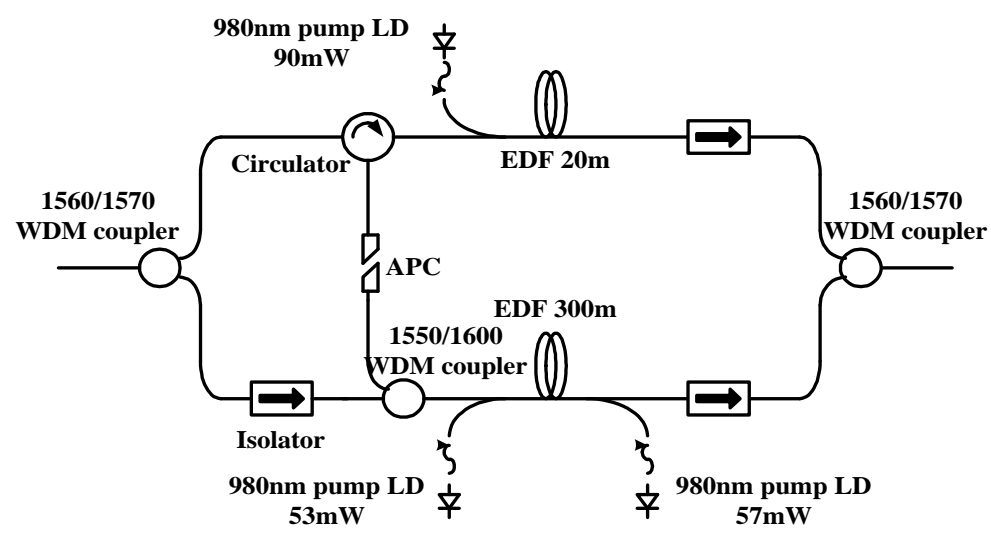

Figure 1-1. Schematics for a coupled, C plus L-band silica based EDFA under study [ref].

Fig. 1-1 shows the coupled structure of C-band plus L-band silica based EDFA used in this study. As an injection source, backward ASE from a C-EDFA was added to an L-EDFA through a circulator and a C/L band WDM coupler. The EDF used in the experiment was a commercially available, Al-codoped one with a peak absorption coefficient of $4.5 \mathrm{~dB} / \mathrm{m}$ at $1530 \mathrm{~nm}$. $980 \mathrm{~nm}$ LDs were used at the power level of $90 \mathrm{~mW}$, $53 \mathrm{~mW}$, and $57 \mathrm{~mW}$, to forward-pump the C-band, forward/backward-pump the L-band EDFA. The measured losses from circulator and C/L WDM coupler were below $0.6 \mathrm{~dB}$ and $0.3 \mathrm{~dB}$. Two external cavity lasers, tuned at 1540 and $1595 \mathrm{~nm}$, were used for the evaluation of the amplifier gain and noise figure, in conjunction with the optical spectrum analyzer.

To compare the performances of the suggested structure with an uncoupled one, we first measured the saturation output power at the signal wavelengths of $1540 \mathrm{~nm}$ and $1595 \mathrm{~nm}$, with C-band backward ASE injection to L-EDFA port being turned off (APC connector open in figure 1-1). The measured signal input / output power for W-EDFA were -3.5dBm / 13.01dBm (@1540nm) and -3.5dBm / 10.47dBm (@1595nm) respectively, exhibiting much lower power conversion efficiency for the L-band. Still, when the seed injection port set in pass-state (APC connector closed), we observed dramatic improvement on the L-EDFA output power over $2.6 \mathrm{~dB}$, with noticeable changes in background ASE spectral profile as well (Figure 1-2). The estimated gain bandwidth of the amplifier from the ASE profile was larger than $80 \mathrm{~nm}$.
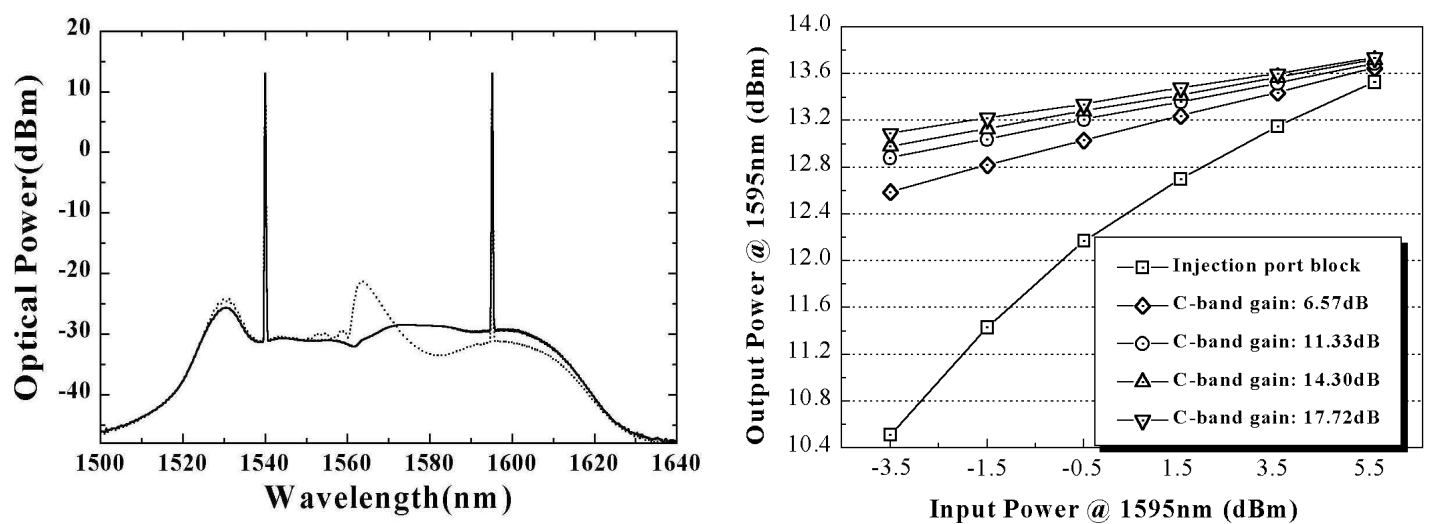

Figure 1-2 (Left). Output spectra of the coupled ( —:solid line) and uncoupled structure ( $\cdots \cdots$ : dot) amplifier for $-3.5 \mathrm{dBm}$ saturating input signals at the wavelengths of $1540 \mathrm{~nm}$ and $1595 \mathrm{~nm}(\mathrm{RBW}=0.2 \mathrm{~nm})$.

Figure 1-3 (Right). Comparison of output powers as a function of $1595 \mathrm{~nm}$ input power at various C-EDFA gain levels. 
To also investigate the possible performance variation of L-EDFA as a function of C-EDFA gain, we measured the saturated output power at the wavelength of $1595 \mathrm{~nm}$, while changing the C-EDFA pump power (Figure 1-3). With the ASE injection port set in pass-state, the L-EDFA exhibited limiting amplifier behavior, with considerable improvement in the gain $(2.1 \sim 2.6 \mathrm{~dB}$ at $-3.5 \mathrm{dBm}$, and $0.1 \sim 0.3 \mathrm{~dB}$ at $6.5 \mathrm{dBm}$ input) and noise figure $(0.6 \mathrm{~dB})$, almost irrespectively of the C-EDFA gain values $(6.57 \sim 17.72 \mathrm{~dB})$. Considering the much smaller relative L-EDFA gain variation below $0.5 \mathrm{~dB}$ for much larger gain change of CEDFA over 11.2dB (and correspondingly different levels of injected C-EDFA backward ASE power), we attribute this behavior to the rapid amplification of the injected C-band ASE signal in the front section of the L-EDFA to a saturation level which then being transferred to L-band photons in rear systems of the amplifier. The above explanation can also be applied to explain the negligible, measured transient crosstalk effect to LEDFA from C-band input power changes. For $6 \mathrm{~dB}$ variation of C-band input power (-4dBm to $2 \mathrm{dBm}$ ), the deviation of L-band output saturating power stayed below $0.2 \mathrm{~dB}$ for wide range of L-EDFA input power levels $(-3.5 \mathrm{dBm} \sim 4.5 \mathrm{dBm})$. Reminding previous results on transient controlled single C-EDFA [6], this CEDFA to L-EDFA crosstalk of $0.2 \mathrm{~dB}$ would not pose a problem for most system applications.

To better understand and confirm the above dynamics / explanation on gain and noise figure improvements, we conducted numerical simulations for the suggested structure. The amplifier was modeled as a homogeneously broadened three level system including both spatial and spectral variations [7], with spectral grid of $1 \mathrm{~nm}$ and spectral range of $120 \mathrm{~nm}$ between $1500 \mathrm{~nm}$ and 1620nm. Absorption and emission cross sections, including other additional parameters were obtained from the fiber manufacturer's data sheets. The simulation results agreed well with the experiments within $1.5 \mathrm{~dB}$ for most spectral range and operating points, and reproduced the general tendency of all the relative performance improvements obtained from the experiments. We attribute the discrepancies in the simulation $(1.5 \mathrm{~dB}$ max for absolute power) to the rather inaccurate component loss profiles and data sets in the long wavelength regime.

The evolution spectra of the forward propagating waves in the L-EDFA sections of the coupled structure and that of the uncoupled structure is shown in Fig 1-4(a) and Fig 1-4(b) respectively. In Fig 1-4(a), the small peak in the shorter wavelength corresponds to the Rayleigh backscattered signal from the C-EDFA, which was included in the simulations. With the injection of C-band backward ASE together with the 980nm pump from the input, 1550nm band seeded forward ASE grows much faster in the input section, when compared to the uncoupled structure. This stronger, amplified forward ASE then get absorbed over the following stages of EDF and transfer its energy to longer wavelengths acting as the primary-pump to L-band photon mediator, while at the same time suppressing the pump-power depleting, wasted backward ASE in the L-EDFA.
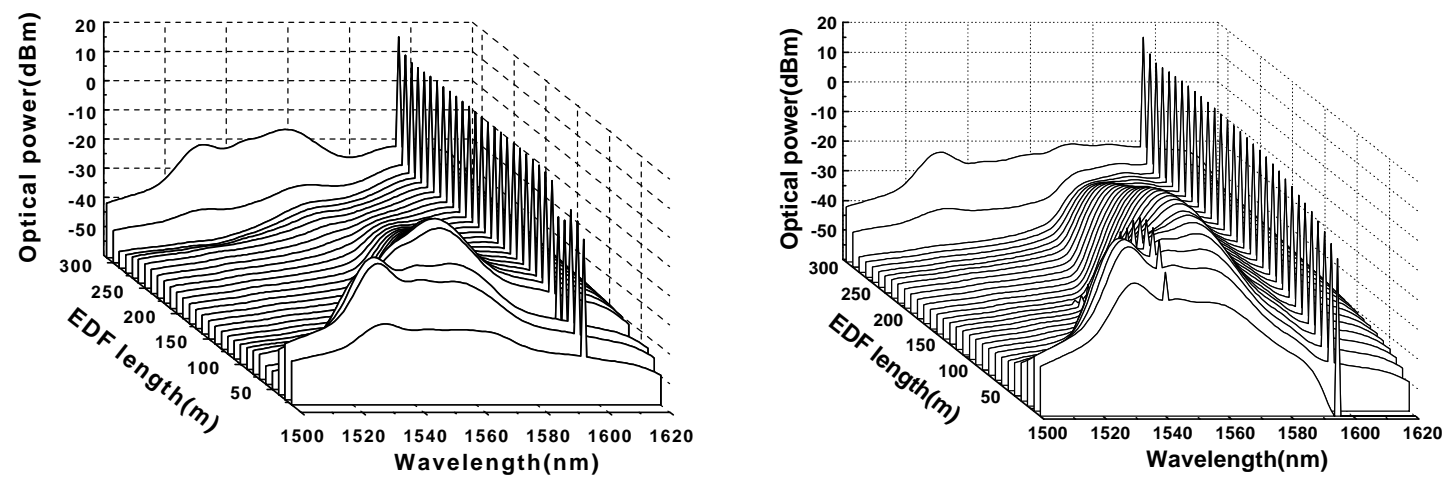

Figure 1-4. Evolution of the forward propagating waves in the L-EDFA sections (a) in the coupled structure (RBW $=1$ $\mathrm{nm})$ and $(\mathrm{b})$ in the uncoupled structure $(\mathrm{RBW}=1 \mathrm{~nm})$

For comparison, the evolution spectrum of the uncoupled W-EDFA exhibits a peculiar dip in powers for forward propagating ASE and signal at about 50m from the input end of L-EDFA. This can be explained by the fact that there are no C-band seed photons in uncoupled structure which mediate the forward 980nm pump to the L-band signals, resulting in the waste of pump power at much faster rate than coupled structure by the strong backward ASE from L-EDFA. Additional distinguished feature can also be found from the evolution 
spectrum of the forward ASE in L-EDFA. In the L-EDFA input sections, ASE is generated mostly in Cband. However, as soon as forward propagating waves diminishes, the newly generated ASE powers after a dip in forward propagating waves are primarily consist of L-band photons. Comparing these two structures, the differences in efficiencies can be attributed to the fact that the seeded photons in the coupled structure act as a reservoir of pump powers for L-band signal amplification.

\subsection{Discussion}

To summarize, we have demonstrated a novel structure for ultra wideband amplifiers with enhanced performances from C-band EDFA backward ASE injection to L-band EDFA, eliminating the need of additional injection sources. Experimental results showed a considerable gain improvement over $2.6 \mathrm{~dB}$ on L-band EDFA, in addition to $0.6 \mathrm{~dB}$ noise figure reduction, without noticeable amount of channel crosstalk from the C-band EDFA to the L-band EDFA. Spatially resolved wideband EDFA simulation were conducted for the first time to our knowledge confirming the experimental observations, and providing better understanding on the dynamics of L-band EDFA.

\section{RAMAN AMPLIFIER MODELING}

\subsection{Introduction}

Raman fiber amplifiers (RFAs) are recently attracting many researcher's attentions in DWDM system application due to their distinctive flexibility in bandwidth designs and growing maturity of high power pump module technologies [8]. However, known modeling techniques for RFAs based on solving ordinary coupled differential equations require exhaustive computational efforts for acquiring well-matched prediction, owing to larger bandwidth and longer fiber length which must be considered in RFAs. In this section, we modify and rearrange the propagation equations of Raman fiber amplifiers so that average power analysis technique can be applied to predictions of system performance with reasonably reduced simulation effort without any degradation of accuracy. Applications of these equations to the numerical analysis of practical RFA-based systems show computation time reduction over 2 orders of magnitude when compared with direct integration approach with comparable accuracy.

\subsection{Theory}

The set of propagation equations governing forward and backward power evolutions in Raman fiber amplifiers which include additional various effects such as Rayleigh backscattering and temperature dependent spontaneous Raman emission can be modeled as $[9,10]$,

$$
\begin{aligned}
& \frac{d P^{ \pm}(z, v)}{d z}= \pm\left\{[-\alpha(v)+A(z, v)-B(z, v)-D(z, v)] P^{ \pm}(z, v)+2 h v \Delta v\left[C(z, v)+\frac{\gamma(v) P^{\mp}(z, v)}{2 h v \Delta v}\right]\right\} \\
& A(z, v)=\sum_{\zeta>v} \frac{g_{r}(v-\zeta)}{K_{e f f}^{A} \text { eff }}\left[P^{ \pm}(z, \zeta)+P^{\mp}(z, \zeta)\right] \\
& B(z, v)=\sum_{\zeta<v} \frac{g_{r}(v-\zeta)}{K_{e f f} A_{e f f}}\left[P^{ \pm}(z, \zeta)+P^{\mp}(z, \zeta)\right] \\
& C(z, v)=\sum_{\zeta>v} \frac{g_{r}(v-\zeta)}{2 A_{e f f}}\left[P^{ \pm}(z, \zeta)+P^{\mp}(z, \zeta)\right]\left[1+\frac{1}{e^{h(\zeta-v) / k T}-1}\right], \\
& D(z, v)=\sum_{\zeta<v} \frac{g_{r}(v-\zeta)}{A_{e f f}}\left[2 h \zeta d \zeta\left\{1+\frac{1}{e^{h(v-\zeta) / k T-1}}\right\}\right]
\end{aligned}
$$


For algebraic simplicity, the power-dependent parameters $A, B, C$ and $D$ were defined as above. The above equations need numerical integration rather than analytical solutions because of the power-dependent $A(z, \mathrm{v})$, $B(z, \mathrm{v}), C(z, \mathrm{v})$ and $D(z, \mathrm{v})$ parameters. However, this analysis problem can be solved with the assumption of the average power analysis technique. First, the length of entire Raman fiber amplifier must be divided into a series of concatenated elemental amplifier sections. Through similar derivation to EDFA analysis [11], when replacing the $A(z, \mathrm{v}), B(z, \mathrm{v}), C(z, \mathrm{v})$ and $D(z, \mathrm{v})$ parameters with constant values $A(\mathrm{v}), B(\mathrm{v}), C(\mathrm{v})$ and $D(v)$, the powers of signals and ASE are substituted by their length averaged powers in each elemental amplifier sections for the calculations of the gain and spontaneous emission. Then iterations are performed until self-consistent convergence condition is satisfied for that section. After these procedures, propagation of powers along the full length of the fiber is continuously modified until the output powers satisfy overall given convergence and boundary conditions through relaxation methods.

\subsection{Numerical Analysis}

As is well known for average power analysis, the simulation results strongly depend on the number of elemental amplifier sections. Fig. 2-1. shows the example case result for the convergence test of the forward and backward power calculated by average power analysis to the result obtained by numerical integration method (using fourth-order Runge-Kutta method with variable step size), as a function of the number of elemental amplifier sections. Though required number of sections is generally dependent on the operating conditions of the Raman fiber amplifier, usually 20 30 sections are sufficient for most system performance evaluations in terms of accuracy. The simulation time was proportional to the number of elemental amplifier sections. By comparison, direct integration method took 228 times more than average power analysis with 20 elemental amplifier sections. Therefore, considerable reduction in computation time as much as 3 orders of magnitude can be obtained for even longer length of Raman fiber amplifiers.

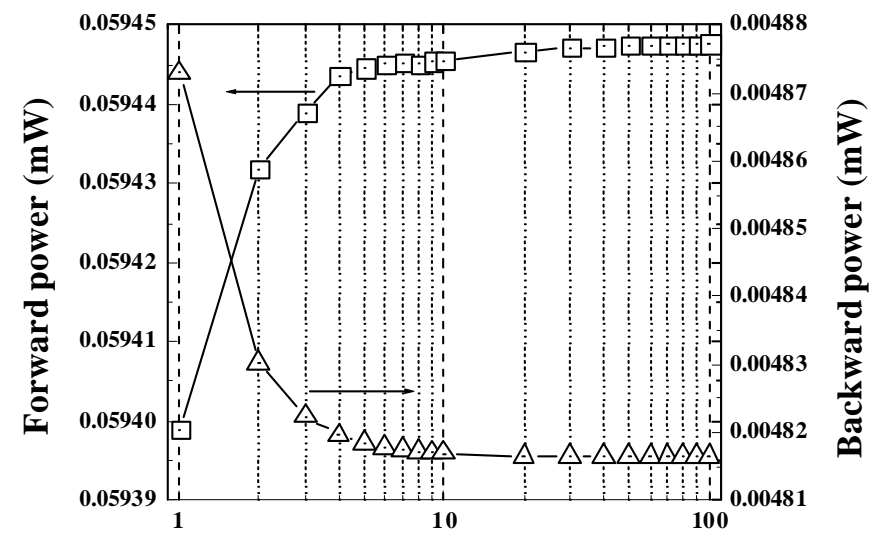

Number of elemental amplifier sections(\#)

Figure 2-1. Forward and backward output powers of RFA as a function of the number of elemental amplifier sections

In order to check the validity of the average power analysis for Raman amplifier, a muti-wavelength pumped distributed Raman amplifier was simulated with the analogous parameter set as previous experimental report [12]. Here, we assumed $25 \mathrm{~km}$ of transmission fiber, 12 backward pump sources (1405, 1412.5, 1420, $1427.5,1435,1442.5,1450,1457.5,1465,1480,1495$ and $1510 \mathrm{~nm})$ and 100 input channels at various power levels. Fig. 2-2. shows the gain and noise figure spectrum of the simulated results according to the various power levels of input signal power. At the signal power levels of $-8 \mathrm{dBm}$, gain deviation less than $1 \mathrm{~dB}$ over $98 \mathrm{~nm}$ is achieved with proper pump power assignments. However, the noise figure spectrum of simulated RFA clearly shows that short wavelength channels near pump bands experience more severe noise accumulation than long wavelength channels. Fig. 2-3. shows the pump evolutions along the length of RFA, exhibiting the strong power transfers between pumps. 

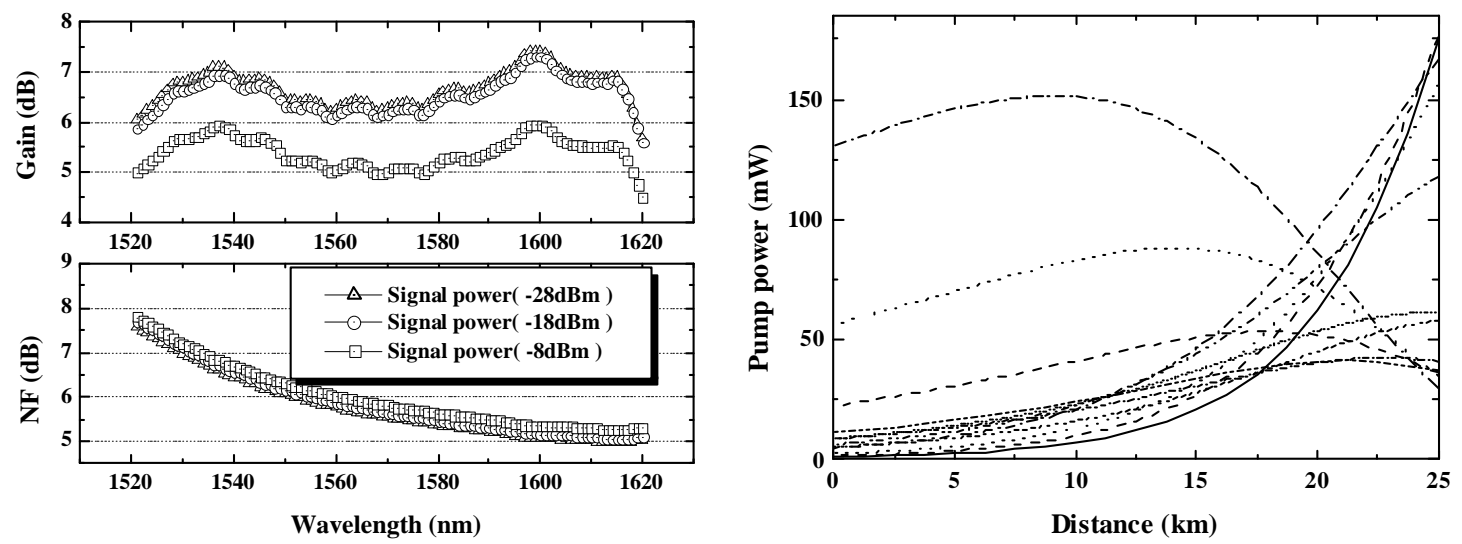

Figure 2-2 (Left). Gain and noise figure of the simulation results at various signal power levels.

Figure 2-3 (Right). Pump power evolutions in the distributed Raman fiber amplifier

\subsection{Discussion}

In summary, we replaced direct integration method of Raman fiber amplifier to relatively simple average power analysis technique and demonstrated its powerful advantages in the required analysis time. Simulation time reduction over 2 orders of magnitude with excellent accuracy promise the future application of this technique to the real situation performance evaluations.

\section{THULIUM AMPLIFIER MODELING}

\subsection{Introduction}

Thulium-doped fluoride fiber amplifiers (TDFFA's) for S+-band (1450-1480 nm) and S-band (1480-1530 $\mathrm{nm}$ ) have been studied extensively in recent years, as the candidates for the next generation amplifiers competing/compromising L-band EDFA's, Raman amplifiers and other rare-earth fiber amplifiers [13-15]. Even though there have been increased experimental reports for $S$ and S+-band TDFFA [16, 17], the complexity of the dynamics from the large number of involving energy levels, and difficulty for measuring experimental parameters from the lack of materials and characterization tools make it very hard to predict the performance of TDFFA. In this section, we present the numerical model for TDFFA, at the same time disclosing the up-conversion pump wavelength dependency for the operation of TDFFA. This model can be also applied to various pumping scheme for $\mathrm{S}$ and $\mathrm{S}^{+}$band. Results show optimum pump wavelength changes (1045-1070nm) as a function of TDFFA pump power / amplifier gains.

\subsection{Theory / Model equations}

The complete set of steady-state model for ZBLAN-based TDFFA has been developed by extending the reduced equation sets of TDFFA and articles on important spectroscopic data on $\mathrm{Tm}^{+}[16,18,19]$. To

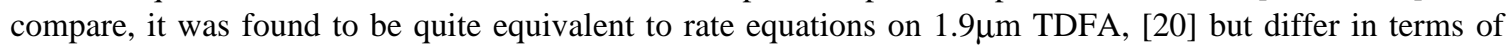
transition levels and other differences resulting from host materials. Included in the rate-equations and propagation-equations were followings: pump ground state absorption (GSA) for ${ }_{3} \mathrm{H}^{6}{ }_{-3} \mathrm{H}^{5}$, pump exited state absorption (ESA) for $\left.{ }_{3} \mathrm{~F}^{4}-{ }_{3} \mathrm{~F}^{2},{ }_{3} \mathrm{~F}^{3}\right)$ and ${ }_{3} \mathrm{H}^{4}{ }_{-1} \mathrm{G}^{4}$, signal stimulated transition for ${ }_{3} \mathrm{~F}^{4}{ }_{3} \mathrm{H}^{4}, 1.8 \mu \mathrm{m}$ band transition for ${ }_{3} \mathrm{H}^{6}{ }_{3} \mathrm{~F}^{4}, 0.8 \mu \mathrm{m}$ band transition for ${ }_{3} \mathrm{H}^{6}{ }_{3} \mathrm{H}^{4}$, and other radiative / non-radiative transitions (Figure 3-1). Within these frames, the equations governing the operation of TDFFA can be written as : 


$$
\begin{aligned}
& \frac{d N_{0}}{d t}=-\left(W_{\mathrm{P} 1}+W_{01}+W_{03}\right) N_{0}+\left(W_{10}+A_{10}+r_{1}\right) N_{1}+A_{20} N_{2}+\left(W_{30}+A_{30}\right) N_{3}+A_{40} N_{4}+A_{50} N_{5} \\
& \frac{d N_{1}}{d t}=W_{01} N_{0}-\left(W_{\mathrm{P} 2}+W_{10}+W_{13}+A_{10}+r_{1}\right) N_{1}+\left(A_{21}+r_{2}\right) N_{2}+\left(W_{31}+A_{31}\right) N_{3}+A_{41} N_{4}+A_{51} N_{5} \\
& \frac{d N_{2}}{d t}=W_{\mathrm{P} 1} N_{0}-\left(A_{21}+A_{20}+r_{2}\right) N_{2}+\left(A_{32}+r_{3}\right) N_{3}+A_{42} N_{4}+A_{52} N_{5} \\
& \frac{d N_{3}}{d t}=W_{03} N_{0}+W_{13} N_{1}-\left(W_{\mathrm{P} 3}+W_{30}+W_{31}+\sum_{\mathrm{i}=0,1,2} A_{3 \mathrm{i}}+r_{3}\right) N_{3}+\left(A_{43}+r_{4}\right) N_{4}+A_{53} N_{5} \\
& \frac{d N_{4}}{d t}=W_{\mathrm{P} 2} N_{1}-\left(\sum_{\mathrm{j}=0,1,2,3} A_{4 \mathrm{j}}+r_{4}\right) N_{4}+\left(A_{54}+r_{54}\right) N_{5} \\
& \frac{d N_{5}}{d t}=W_{\mathrm{P} 3} N_{3}-\left(\sum_{\mathrm{k}=0,1,2,3,4} A_{5 \mathrm{k}}+r_{5}\right) N_{5} \\
& N=N_{0}+N_{1}+N_{2}+N_{3}+N_{4}+N_{5} \\
& \text { where } W_{i j}=\int_{\ddot{\mathrm{e}}} \frac{\Gamma(\ddot{\mathrm{e}}) \sigma_{i j}^{e}(\ddot{\mathrm{e}}) P(\ddot{\mathrm{e}})}{h v A_{e f f}(\ddot{\mathrm{e}})} d \ddot{\mathrm{e}} \\
& \left.\frac{d P^{ \pm}(\lambda)}{d z}= \pm P(\lambda)^{ \pm} \Gamma(\lambda)\left(\sum_{(\mathrm{j}, \mathrm{k})}^{(1,0),(3,0),(3,1)} \sigma_{\mathrm{jk}}^{e}(\lambda) N_{\mathrm{j}}-\sigma_{\mathrm{kj}}^{a}(\lambda) N_{\mathrm{k}}\right)-\sigma_{\mathrm{P} 1}^{a}(\lambda) N_{0}-\sigma_{\mathrm{P} 2}^{a}(\lambda) N_{1}-\sigma_{\mathrm{P} 3}^{a}(\lambda) N_{3}\right) \\
& \pm 2 h v \Delta v \Gamma(\lambda)\left(\sum_{\mathrm{i}=1,3} \sigma_{\mathrm{i} 0}^{e}(\lambda) N_{\mathrm{i}}+\sigma_{31}^{e}(\lambda) N_{3}\right) \mp \alpha(\lambda) P^{ \pm}(\lambda)
\end{aligned}
$$
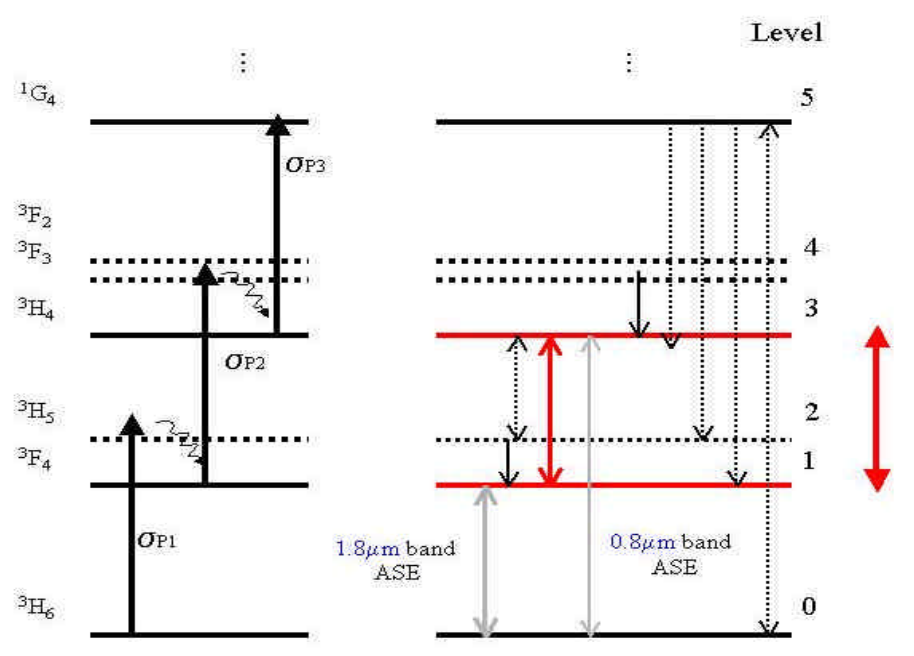

Figure 3-1. Energy level diagram and : (a) pump transition process, (b) other radiative and non-radiative transitions. 
where, for each energy level i and $\mathrm{j}$, Ni denotes the population of level i, $\mathrm{N}$ the total number of Tm $3+$ ions per $\mathrm{cm} 3$, Wij the transition rate of absorption $(\mathrm{i}<\mathrm{j})$ and emission $(\mathrm{i}>\mathrm{j})$, Wp the pump absorption rate, oij crosssection, Aij spontaneous emission probability, ri multi-phonon decay rate, $\Gamma$ overlap factor, and $\alpha$ fiber background loss, the + , - sign the direction of propagation forward and backward, respectively. In the model, the pump GSA and pump ESA, as well as other relevant transition sets were included to fully analyze additional effects on signal transitions between $3 \mathrm{H} 4-3 \mathrm{~F} 4$ band. In this way, it was possible to include the effects from the signal transitions between the excited levels of Tm3+ (which differ from Er3+) and selfterminating nature of signal band. This involves many transition processes simultaneously over the wide wavelength window.

Fig. 3-1 shows the levels for the atomic system and the related transitions for model for pump transition processes (a) and other radiative and non-radiative processes (b). The ground state absorption (GSA) $\sigma \mathrm{p} 1$ and excited state absorption (ESA) $\sigma \mathrm{p} 2$ are shown in Fig. 3-2. The parameter sets for the simulation were carefully chosen mostly from the published data, except some unpublished secondary parameters which were then indirectly acquired from the theoretical analysis based on past reports on Tm3+. Lifetimes and branching ratios given by Judd-Ofelt theory, multi-phonon decay rate by energy gap law, and the previously reported cross-sectional data are also included in the model. The mode parameters such as index difference and core diameter are also has been included. The constructed model showed reasonably matching results (in the order of a dB) when compared to formerly published experiments under various operating conditions $[16,21,22]$, validating our successful construction of the TDFFA simulator.
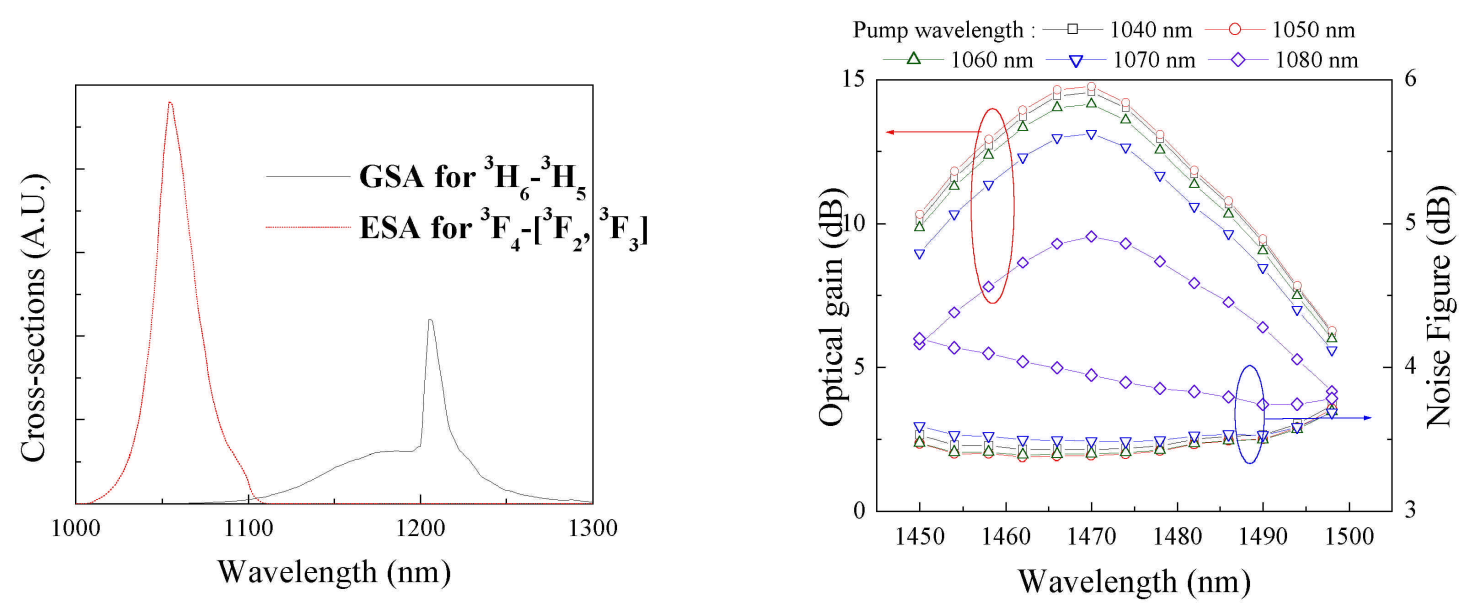

Figure 3-2. (Left) Pump ground state absorption (GSA) op1, \& pump excited state absorption (ESA) $\sigma p 2$ [4,6](estimated)

Figure 3-3. (Right) Gain and NF spectra for WDM input at various pump wavelengths with $-20 \mathrm{dBm}$ channel power and forward pump power of $150 \mathrm{~mW}$

\subsection{Numerical Analysis}

To investigate the pump wavelength dependency, we constructed a model TDFA by using a 10 m's of TDF with 1000 ppm of Tm3+ doping concentration [16]. Assuming WDM applications, fig. 3 shows the gain and noise figure (NF) spectra for $13 \mathrm{WDM}$ channels with $-20 \mathrm{dBm}$ channel input, ranging from $1450 \mathrm{~nm}$ to $1498 \mathrm{~nm}$, as a function of pump wavelength from $1040 \mathrm{~nm}$ to $1080 \mathrm{~nm}$ at the fixed forward pumping power of $150 \mathrm{~mW}$. Power conversion efficiency (PCE) improvement was observed near the pump wavelength of 1045 $\mathrm{nm}$. For longer pump wavelengths, dropped peak-gain and broadened 3dB-bandwidth has been observed at the same pump power. Noise figure (NF) values for these cases are also shown in Fig. 3-3, exhibiting ignorable dependency on the pumping wavelengths. 
Fig. 3-4 (a) shows the peak channel gain as a function of pumping wavelength, each obtained at different forward pumping powers. The optimum pump wavelength was near $1070 \mathrm{~nm}$ for the weak pump regime and moved to $1045 \mathrm{~nm}$ as the value of pumping power increases. The locus of optimum pump wavelengths at peak gain values is also plotted in Fig. 3-4(a). Figure 3-4(b) shows peak gain value as a function of pump power under different pump wavelengths. We attribute the shift of optimum pump wavelength to the GSA cross-section $\sigma \mathrm{p} 1$ (peak at $1210 \mathrm{~nm}$ ), which has a destructive contribution to inversion (especially in the high pump regime) combined with the constructive effect for the increase of $\sigma \mathrm{p} 2$ (peak at $1055 \mathrm{~nm}$ ).
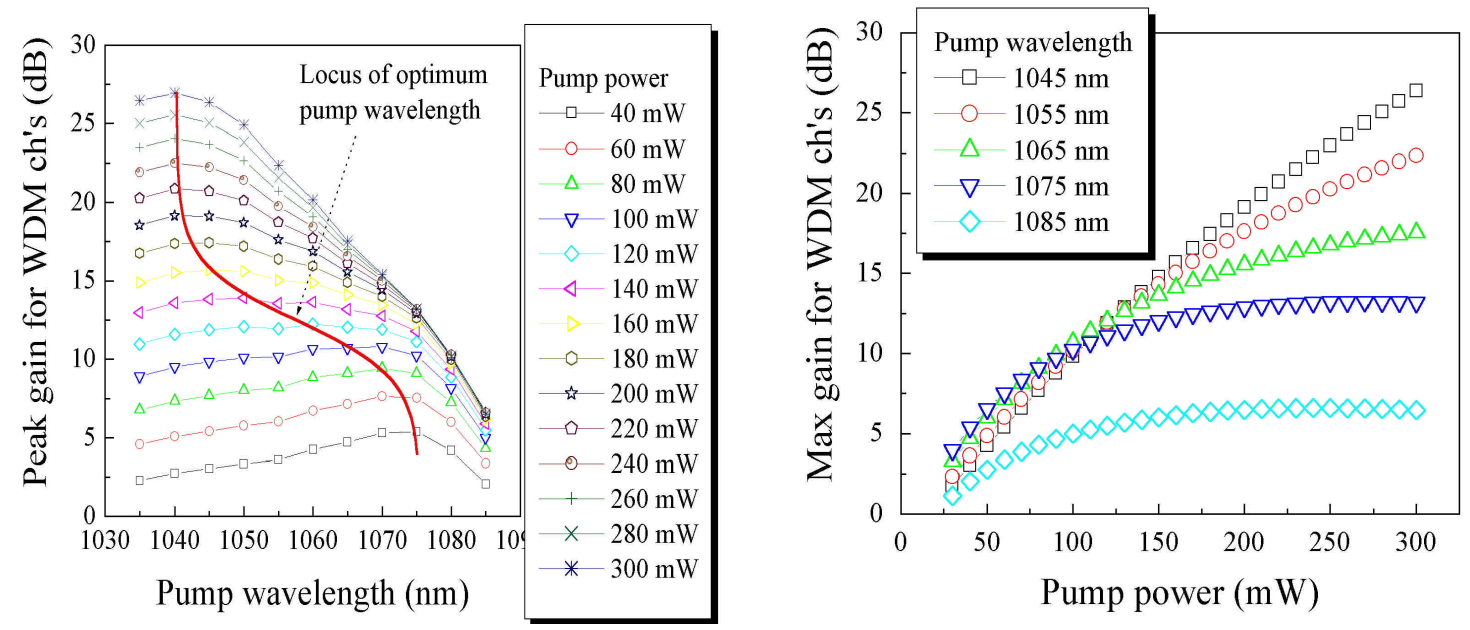

Figure 3-4. Peak gain for WDM input versus (a) pump wavelength from $40 \mathrm{~mW}$ to $300 \mathrm{~mW}$ pump power, and (b) pump power under various pump wavelength.

\subsection{Discussion}

In this section, we report a successful full-set numerical model for TDFA, and investigate the pump wavelength dependency of TDFA performances. The optimum pump wavelength shift toward near $1045 \mathrm{~nm}$ from $1070 \mathrm{~nm}$ as pumping power increase. Thus the usage of conventional $1064 \mathrm{~nm}$ pump may lead to a penalty on PCE in high pumping regime. This result could be used for the practical design and prediction for the performance of TDFA as one of the next generation amplifiers.

\section{SUMMARY}

In this paper, we presented numerical analysis result for three different types of amplifiers, which have different amplification dynamics and gain bands. Applications of numerical analysis to subjects of interest were carried out either to grab useful insights of the inner physics, or to prove the algorithm of the numerical methods under harsh test conditions. Even the modeling of each amplifier presented in individual sections, the integration of these model equations for the investigation of hybrid amplifiers should be straightforward. Numerical analyses for transient phenomena in the amplifiers, which require average inversion model with time dependent equations, were not included in this paper but the implementation should be straightforward for EDFA and Raman amplifiers. Also excluded in the discussion is the black box modeling, often useful in predicting the amplifier performance from externally measured experimental data sets. In the numerical analysis regime, these wavelength domain data obtained with above techniques described in the manuscript can be further applied to the time domain simulation tools to get correct $\mathrm{Q}$ factors and to estimate the power margin / BER for the target transmission system designs. 


\section{REFERENCES}

1. M. Yamada, H. Ono, T. Kanamori, S. Sudo, and Y. Ohishi, "Broadband and gain-flattened amplifier composed of a $1.55 \mu \mathrm{m}$-band and a $1.58 \mu \mathrm{m}$-band Er3+-doped fiber amplifier in a parallel configuration," Electron. Lett., vol. 33, no. 8, pp. 710-711, 1997.

2. Y. Sun, J. W. Sulhoff, A. K. Srivastava, J. L. Zyskind, T. A. Strasser, J. R. Pedrazzani, C. Wolf, J. Zhou, J. B. Judkins, R. P. Espindola, and A. M. Vengsarkar, "80 nm ultra-wideband erbium-doped silica fiber amplifier,” Electron. Lett., vol. 33, no. 23 , pp.1965-1967, 1997

3. J. Nilsson, S. Y. Yun, S. T. Hwang, J. M. Kim, and S. J. Kim, "Long-wavelength erbium-doped fiber amplifier gain enhanced by ASE end-reflectors," IEEE Photon. Technol. Letter., vol. 10, no. 11, pp. 1551-1553, 1998

4. J. Lee, U. C. Ryu, S. J. Ahn, and N. Park, "Enhancement of power conversion efficiency for an L-band EDFA with a secondary pumping effect in the unpumped EDF section," IEEE Photon. Technol. Letter., vol. 11, no. 1, pp. 42-44, 1999

5. Y. Sugaya, S. Kinoshita, M. Takeda, C. Ohshima, and T. Chikama, "1.58 $\mu \mathrm{m}$ band Er3+-doped fibre amplification with a 1.55 $\mu$ m-band light injection ”, OECC' 98 Tech. Dig., pp.498-499, paper 16C2-4.

6. H. Yoon, S. Bae, S. J. Ahn, and N. Park, "Reference level free multichannel gain equalization and transient gain suppression of EDFA with differential ASE power monitoring," IEEE Photon. Technol. Letter., vol. 11, no. 3, pp. 1-3, 1999

7. E. Desurvire, Erbium Doped Fiber Amplifier; Principles and Applications. New York: Wiley, 1994.

8. S. V. Chernikov, S. A. E. Lewis, and J. R. Taylor, "Broadband Raman amplifiers in the spectral range of 1480-1620 nm,” in OFC' 99 Tech. Dig., 1999, pp. 117-119.

9. H. Kidorf, K. Rottwitt, M. Nissov, M. Ma, and E. Rabarijaona, "Pump interactions in a 100-nm bandwidth Raman amplifier,” IEEE Photon.Technol. Lett., vol. 11, pp. 530-532, May 1999.

10. W. A. Reed, W. M. Coughran, and S. G. Grubb, "Numerical modeling of cascaded CW Raman fiber amplifiers and lasers," AT\&T Bell Labo-ratories, Tech. Memo., 1994.

11. T. G. Hodgkinson, "Improved average power analysis technique for er-bium-doped fiber amplifiers," IEEE Photon. Technol. Lett., vol. 4, pp. 1273-1275, Nov. 1992.

12. Y. Emori and S. Namiki, "100-nm bandwidth flat-gain Raman ampli-fiers pumped and gain-equalized by 12-wavelength-channel WDM high power laser diodes,” OFC' 99 Tech. Dig., 1999, pp. PD19-1-PD19-3.

13. Bumki Min, Hosung Yoon, Won Jae Lee, and Namkyoo Park, "Coupled Structure for Wideband EDFA with Gain and Noise Figure Improvement from C to L-band ASE Injection,” IEEE Photon. Technol. Lett., vol. 12, pp. 480-482, May 2000.

14. J. Kani, M. Jinno, "Wideband and flat-gain optical amplification from 1460 to $1510 \mathrm{~nm}$ by serial combination of a thulium-doped fluoride fibre amplifier and fibre Raman amplifier," Electron. Lett., vol. 35, pp. 1004-1006, 1999.

15. Bumki Min, Won Jae Lee, and Namkyoo Park, "Efficient Formulation of Raman Amplifier Propagation Equations with Average Power Analysis," Photon. Technol. Lett,.vol. 12, no. 11, pp.1486-1488, 2000

16. T. Komukai, T. Yamamoto, T. Sugawa, and Y. Miyajima, "Upconversion pumped thulium-doped fluoride fiber amplifier and laser operating at $1.47 \mu \mathrm{m}$," IEEE J. Quantum Electron., vol. 31, no. 11, pp. 1880-1889, 1995.

17. T. Kasamatsu, Y. Yano, and H. Sekita, "Novel 1.50- $\mu \mathrm{m}$ band gain-shifted thulium-doped fiber amplifier by using dual wavelength pumping of $1.05 \mu \mathrm{m}$ and $1.56 \mu \mathrm{m}, "$ Proc. OAA'99, PDP1, 1999.

18. M. J. F. Digonnet, Ed., Rare earth doped fiber lasers and amplifiers, Marcel Dekker, New York, USA, 1993, chapter 2, 10.

19. J. Sanz, R. Cases, and R. Alcala, "Optical properties of Tm3+ in fluorozirconate glass," J. Non-crystal. Solids, vol. 93, pp 377-386, 1987.

20. S. D. Jackson, T. A. King, "Theoretical modeling of Tm-doped silica fiber lasers," J. Lightwave Technol., vol. 17, pp. 948-956. 1999.

21. T. Sakamoto, S. Aozasa, T. Kanamori, K.Hoshino, M. Yamada, M. Shimizu, "Gain-equalized thulium doped fiber amplifiers for 1460 nm-band WDM signals," Proc. OAA'99, Nara, Japan, WD2, 1999.

22. T. Sakamoto, M. Yamada, M.Shimizu, T. Kanamori, Y. Terunuma, Y. Ohishi, S.Sudo, "Thulium-doped fluoride amplifiers for $1.4 \mu \mathrm{m}$ and $1.6 \mu \mathrm{m}$ operation," Proc. OAA'96, Monterey, CA, USA, ThC3, 1996. 